\title{
Smoking: additional burden on aging and death
}

\author{
Masahiko Watanabe
}

\begin{abstract}
Tobacco smoking is a major cause of lung cancer. It has been suggested that there is an approximately linear dose-response relationship between the number of cigarettes smoked per day and clinical outcome such as lung cancer mortality. It has also been proposed that there is a greater increase in mortality at high doses when the dose is represented by the duration of the smoking habit rather than the number of cigarettes. The multistep carcinogenesis theory indicates that a greater increase in mortality rate at high doses is possible, as is the case between aging and cancer, even though each dose-response relationship between a carcinogenic factor and a carcinogenic step forward is linear. The high incidence of lung cancer after long-term smoking and the decreased relative risk after smoking cessation suggests a similarity between the effects of smoking and aging. Prediction of lung cancer risk in former smokers by simple integration of smoking effects with aging demonstrated a good correlation with that estimated from the relative risk of the period of smoking cessation. In contrast to the smoking period, there appears to be a linear relationship between smoking strength and cancer risk. This might arise if the dose-response relationship between smoking strength and each carcinogenic step is less than linear, or the effects become saturated with a large dose of daily smoking. Such a dose-response relationship could lead to relatively large clinical effects, such as cardiovascular mortality, by low-dose tobacco smoke exposure, e.g., second-hand smoking. Consideration of the dose-response of each effect is important to evaluate the risk arising from each carcinogenic factor.
\end{abstract}

Keywords: Cigarette smoking, Smoking cessation, Dose-response relationship, Aging, Cancer risk

\section{Introduction - smoking as a human carcinogen}

Smoking causes a variety of diseases including cancer. In 1981, Doll and Peto estimated that $30 \%$ of all cancer deaths were attributable to tobacco smoking [1]. This estimation is basically similar in Japan, as the population attributable fractions of cancer mortality related to tobacco smoking in Japanese men and women in 2005 have been estimated to be $34.4 \%$ and $6.2 \%$, respectively [2]. Tobacco smoke contains many kinds of carcinogens. The International Agency for Research on Cancer evaluated and classified 16 compounds present in tobacco smoke as Group 1 - carcinogenic to humans [3]. The tumor promotion activity of tobacco smoke is also established $[3,4]$.

Correspondence: wa1@shujitsu.ac.jp

School of Pharmacy, Shujitsu University, 1-6-1 Nishigawara, Okayama 703-8516, Japan

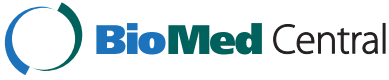

\section{Relationship between dose-response of each carcinogenic step and clinical outcome}

The simplest model to estimate the clinical consequence of a carcinogenic factor, or dose-response relationship between the factor such as tobacco smoking and cancer mortality, is linear-no-threshold, while cancer emerges after progressing through a number of steps in the carcinogenic process $[5,6]$. When the dose-response relationship between a factor and each carcinogenic step, such as initiation by mutation induction, is linear, the clinical response is also linear at low doses, called "linearized multistage" [7]. This holds true regardless of whether the factor affects a single or a number of carcinogenic steps. However, this prediction is not applicable at high doses. When the factor affects many carcinogenic steps such as mutation induction and cell growth, the clinical dose-response relationship is more than linear, or a steeper increase in cancer incidence with the increment in dose occurs, even when the dose- 
response relationship for each step is linear. It is indeed a case of the factor "aging", since cancer mortality generally increases almost logarithmically with increasing age.

In addition to the traditional Armitage-Doll multistage model, there are many mathematical models to explain the relationship between cancer incidence and age [8-13]. For smoking and smoking-related cancers such as lung cancer, approximately linear dose-response relationships have been observed in a number of epidemiological studies when the dose was represented by the number of cigarettes per day [14-19]. When the dose was represented by the duration of the smoking habit, a greater than linear increment in incidence or mortality was reported [20-22]. Because a life-long smoking habit can be considered as a very high dose of carcinogenic factor, it is possible that the duration of the smoking habit affects several steps of carcinogenesis in a linear relationship, but causes a higher incidence of cancer after long-term smoking. Note that, if a factor affects only one step in a linear dose-response relationship rather than multiple steps described above, the incidence of cancer at a high dose is dependent on which step is affected and the mechanism of cancer induction, such that both a steep increment or a plateau are possible.

\section{Smoking as an aging accelerator}

Naturally, cancer occurs during aging because activation of steps in the carcinogenic process, such as mutation induction and cell growth, increase during aging. Tobacco smoking exhibits a tumor promoting effect as well as induction of mutation, thus smoking induces a number of steps in carcinogenesis. Therefore, when smokers stop smoking, the relative risk of developing cancer gradually decreases compared with the risk in continuing smokers, but it does not fall to the level of nonsmokers, since past smoking continues to have an effect even after long-time smoking cessation. Most studies have demonstrated that the risk in former smokers is far lower than in continuing smokers but remains higher than that in nonsmokers [15, 20-24]. There are several reports concerning cancer risk prediction, from simple approximation by regression equation to rather complicated approaches, including validation of several mathematical models [25-29]. If smoking affects all carcinogenic steps in the same proportion to that of aging, the effects of smoking can be simplified as an additional aging effect, and risk prediction in continuing and former smokers may be possible regardless of the cancer model. I examined such a simple explanation of smoking effects using data reported by Peto et al. [20].

In considering lung cancer death, probability is assumed to be determined by aging level $x+a(s-l)$, where $x, s, l$, and $a$ are age, smoking period, lag time, and coefficient of aging effect by smoking. The parameters $a$ and
Table 1 Age and cumulative lung cancer mortality of continuing and former smokers

\begin{tabular}{lll}
\hline Age (age at cessation) & Mortality $(\%)^{a}$ & Age corresponding to smoker \\
\hline Continuing smoker & & \\
50 & 0.40 & \\
55 & 0.95 & \\
60 & 2.25 & \\
65 & 5.00 & \\
70 & 9.65 & \\
75 & 15.9 & \\
Former smoker & & \\
75 (60) & 9.9 & 70.22 \\
75 (50) & 6.0 & 66.27 \\
75 (40) & 3.0 & 61.72 \\
$75(30)$ & 1.7 & 58.37 \\
\hline
\end{tabular}

${ }^{\mathrm{a}}$ Data from Doll et al. [20]

${ }^{\text {b} T h e ~ c o r r e s p o n d i n g ~ c o n t i n u i n g ~ s m o k e r ' s ~ a g e ~ a t ~ w h i c h ~ c u m u l a t i v e ~ l u n g ~ c a n c e r ~}$ mortality risk is equal to the risk of that in former smokers at age 75 was calculated from the quintic equation ${ }^{c}$ which takes account of the risk in continuing smokers. It is a simple technique and not dependent on the cancer model. The difference between age 75 and the corresponding age is considered to be "delayed effect on aging" by cessation of smoking (Fig. 1)

$c y=-1.333333 \times 10^{-6} x^{5}+3.833333 \times 10^{-4} x^{4}-0.04306667 x^{3}+2.381417 x^{2}-$ $65.0675 x+704.4$

$l$ were estimated from the former smoker's age corresponding to that of a continuing smoker (Table 1 and Fig. 1). When the risk of nonsmokers and smokers were approximated by a single polynomial equation, the highest correlation was observed if smoking started at 11 years old (Fig. 2). This estimation suggested that smoking effects would be stronger at younger ages, possibly because the smoke would induce mutations at relatively higher levels than promotion of cell growth, compared with older age.

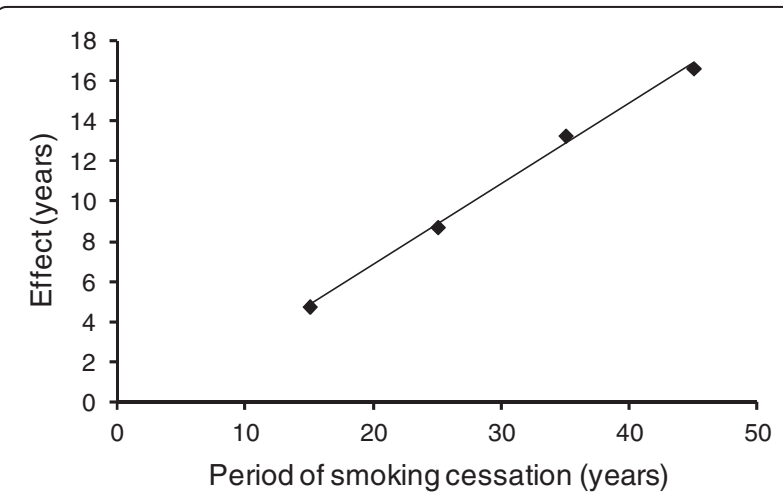

Fig. 1 Estimation of the aging effects of smoking. Prolonged smoking cessation resulted in a decreased relative risk of lung cancer mortality, as exhibited by the "delayed effect on aging" (Table 1). The relationship between the period of smoking cessation and the effect was plotted. Linear regression yielded the equation $y=0.401 x-1.175$. The coefficient of the aging effect of smoking was $0.401 /(1-0.401)=0.669$. This means that smokers become old 1.669 times faster than nonsmokers. The lag time between smoking and death was $1.175 / 0.401=2.93$ years 


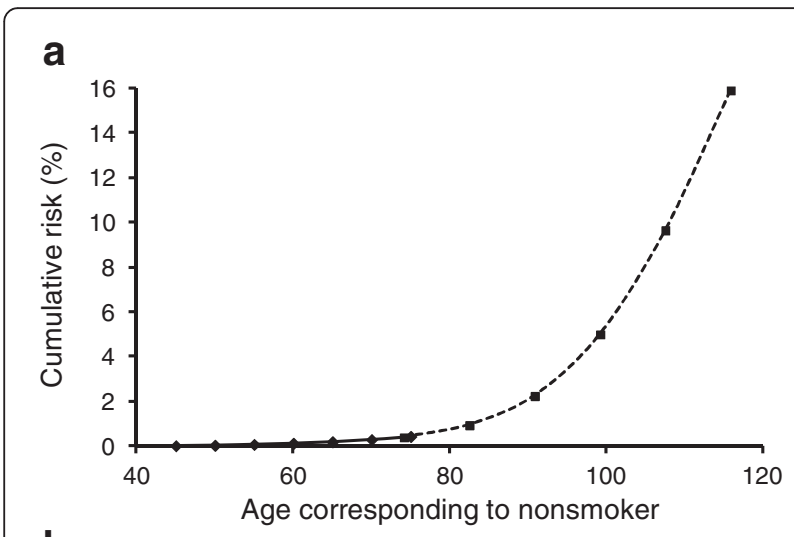

b

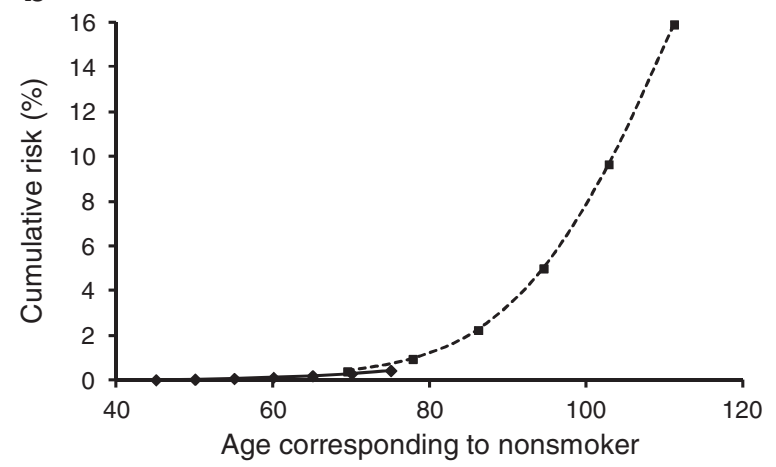

Fig. 2 Cumulative lung cancer mortality risk of a nonsmoker and continuing smoker. Data of risk were from Doll et al [20]. The continuing smoker's age corresponding to that of a nonsmoker was calculated from the coefficient of the aging effect and the lag time (Fig. 1), and age at starting smoking. a Smoking started at age 11; b Smoking started at age 18. Solid line, nonsmoker; dashed line, continuing smoker. It should be noted that these figures demonstrate the smoking effect as additional aging effect during the smoking period and do not predict the risk in a very old person. It is known that cancer risk in the very old is often lower than that predicted by various cancer models. The sextic regression equation obtained from the risk in a nonsmoker and continuing smoker starting at age 18 together was: $y=-5.096171 \times 10^{-10} x^{6}+1.983542 \times 10^{-7} x^{5}-3.006177 \times$ $10^{-5} x^{4}+2.309032 \times 10^{-3} x^{3}-0.0953864 x^{2}+2.014889 x-17.00096$

A good correlation was still observed by assuming a higher starting age, and thus the age-specific cumulative risks of former smokers were calculated using the equation and assuming a starting age of 18 years (Fig. 3). This risk estimation accorded well with that estimated from the relative risk of the period of smoking cessation [20]. Hence, the effects of the smoking habit may be roughly approximated as an aging effect. Although the data used in this study were from case-control studies combined with national statistics [20], the relative risk of former smokers according to the period of smoking cessation in a variety of large cohort studies in the United States [24] was similar to that of the case-control studies.

A report which analyzed three cohort studies in Japanese subjects suggested that the risk of lung cancer in former smokers decreased to the level in nonsmokers

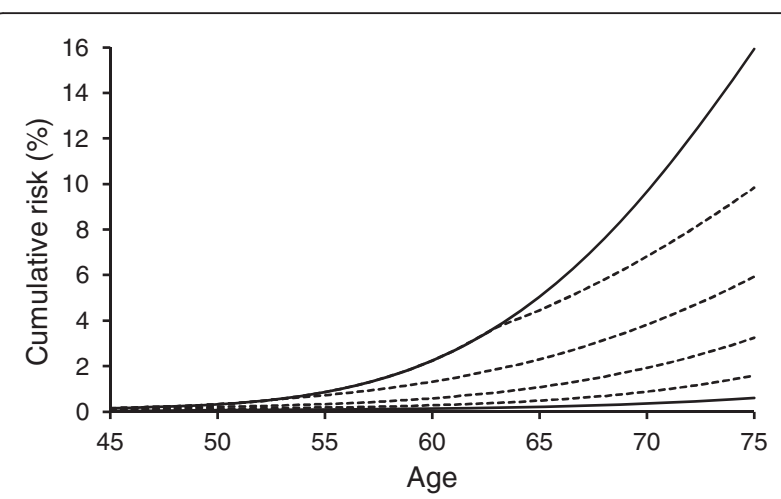

Fig. 3 Effect of smoking cessation at various ages on the cumulative lung cancer mortality risk. A single polynomial regression equation (Fig. 2) and age corresponding to a nonsmoker was used. The age of starting smoking was assumed to be 18. The upper and lower solid lines are the risks in continuing smokers and nonsmokers, respectively. The dashed lines are the risks in former smokers who stopped at age 60, 50, 40, and 30 years, from the higher to lower lines

after a long period of smoking cessation [30]. The reason is unclear, but the relative risk of lung cancer in Japanese subjects was lower than that in Caucasians [31, 32], and the detection power was also lower. In addition, the scale of the study was smaller than the cohort studies in Caucasians, thus it is possible that a small difference in risk between long-term quitters and nonsmokers could not be detected.

\section{Risk estimation - lesson from the smoking effect}

When dose is represented by the number of cigarettes per day rather than the duration of the smoking period, approximately linear dose-response relationships are observed in most studies [14-19]. This is in contrast to the effect of smoking duration or aging. From the observation, it is possible that a dose-response relationship between smoking strength and each carcinogenic step, such as initiation and promotion of cancer, is less than linear, or the effects become saturated by a large amount of daily smoking. Using data of large epidemiological studies, the British Doctor Study, the American Cancer Society's Cancer Prevention Study I and II, the Nurses Health Study and the Health Professionals Follow-Up Study in a two-stage clonal expansion model, the promotion rate powers were estimated to be around 0.5 $[28,29]$. This means that the effects of smoking per dose are relatively stronger at lower doses, i.e., second-hand smoking or light smoking. The model simulation provides a different concept from that which suggests there is a threshold for indirect carcinogens. However, it is known that the effects of air pollution and second-hand smoking on cardiovascular mortality per exposure dose are higher than that of active smoking, which has an apparently higher exposure dose [16, 33]. Adverse effects 
on the cardiovascular system such as oxidative stress, vascular inflammation and dysfunction, activation of the hemostatic system, and disturbance of autonomic nervous system [34, 35], are suggested to be relatively stronger at low doses. In addition, the relative effect of second-hand smoking on lung cancer induction may also be higher than that of active smoking $[16,36]$. Therefore, the effects of smoking, such as cancer promotion at low doses may be stronger per dose than at high doses. This case clearly demonstrates the need for careful examination of linear-no-threshold extrapolation as the concept of the "safe side" of risk estimation.

\section{Conclusion}

Smoking is an additional burden on aging and death. It is simple and useful approach to estimate lung cancer risk from duration of smoking habit. Consideration of the dose-response of each effect is important to evaluate the risk arising from each carcinogenic factor including tobacco smoke.

\section{Competing interest}

The author declares that no conflicts of interest exist.

\section{Acknowledgments}

The author thanks Dr. Shinji Oikawa and Dr. Hiroyuki Kamiya, Organizer of 2015 JEMS Symposium, and the Japanese Environmental Mutagen Society.

Received: 13 October 2015 Accepted: 4 December 2015

Published online: 22 January 2016

\section{References}

1. Doll R, Peto R. The causes of cancer: quantitative estimates of avoidable risks of cancer in the United States today. J Natl Cancer Inst. 1981;66:1191-308.

2. Inoue M, Sawada N, Matsuda T, Iwasaki M, Sasazuki S, Shimazu T, et al. Attributable causes of cancer in Japan in 2005-systematic assessment to estimate current burden of cancer attributable to known preventable risk factors in Japan. Ann Oncol. 2012;23:1362-9.

3. IARC. Tobacco smoke and involuntary smoking. IARC Monogr Eval Carcinog Risks Hum. 2004;83:1-1418.

4. IARC. Tobacco smoking. IARC Monogr Eval Carcinog Risks Hum 2012;100E:43-211.

5. Kinzler KW, Vogelstein B. Lessons from hereditary colorectal cancer. Cell. 1996;87:159-70

6. Hanahan D, Weinberg RA. The Hallmarks of Cancer. Cell. 2000;100:57-70

7. Guess H, Crump K, Peto R. Uncertainty estimates for low-dose-rate extrapolations of animal carcinogenicity data. Cancer Res. 1977;37:3475-83.

8. Armitage P, Doll R. The age distribution of cancer and a multi-stage theory of carcinogenesis. Br J Cancer. 1954;8:1-12.

9. Moolgavkar SH, Venzon DJ. Two-event models for carcinogenesis: incidence curves for childhood and adult tumors. Math Biosci. 1979;47:55-77.

10. Luebeck EG, Moolgavkar SH. Multistage carcinogenesis and the incidence of colorectal cancer. Proc Natl Acad Sci U S A. 2002;99:15095-100.

11. Michor F, Iwasa Y, Nowak MA. The age incidence of chronic myeloid leukemia can be explained by a one-mutation model. Proc Natl Acad Sci U S A. 2006;103:14931-4.

12. Little MP. Cancer models, genomic instability and somatic cellular Darwinian evolution. Biol Direct. 2010;5:19.

13. Luebeck EG, Curtius K, Jeon J, Hazelton WD. Impact of tumor progression on cancer incidence curves. Cancer Res. 2013;73:1086-96.

14. Doll R, Peto R, Boreham J, Sutherland I. Mortality in relation to smoking: 50 years' observations on male British doctors. BMJ. 2004;328:1519.

15. Kenfield SA, Stampfer MJ, Rosner BA, Colditz GA. Smoking and smoking cessation in relation to mortality in women. JAMA. 2008;299:2037-47.
16. Pope III CA, Burnett RT, Turner MC, Cohen A, Krewski D, Jerrett M, et al. Lung cancer and cardiovascular disease mortality associated with ambient air pollution and cigarette smoke: shape of the exposure-response relationships. Environ Health Perspect. 2011:119:1616-21.

17. Pirie K, Peto R, Reeves GK, Green J, Beral V. The 21st century hazards of smoking and benefits of stopping: a prospective study of one million women in the UK. Lancet. 2013;381:133-41.

18. Akiba S, Hirayama T. Cigarette smoking and cancer mortality risk in Japanese men and women-results from reanalysis of the Six-Prefecture Cohort Study Data. Environ Health Perspect. 1990;87:19-26.

19. Ando M, Wakai K, Seki N, Tamakoshi A, Suzuki K, Ito Y, et al. Attributable and absolute risk of lung cancer death by smoking status: findings from the Japan Collaborative Cohort Study. Int J Cancer. 2003;105:249-54.

20. Peto R, Darby S, Deo H, Silcocks P, Whitley E, Doll R. Smoking, smoking cessation, and lung cancer in the UK since 1950: combination of national statistics with two case-control studies. BMJ. 2000;321:323-9.

21. Brennan P, Crispo A, Zaridze D, Szeszenia-Dabrowska N, Rudnai P, Lissowska J, et al. High cumulative risk of lung cancer death among smokers and nonsmokers in Central and Eastern Europe. Am J Epidemiol. 2006;164:1233-41.

22. Bosetti C, Gallus S, Peto R, Negri E, Talamini R, Tavani A, et al. Tobacco smoking, smoking cessation, and cumulative risk of upper aerodigestive tract cancers. Am J Epidemiol. 2008:167:468-73.

23. Lee YC, Marron M, Benhamou S, Bouchardy C, Ahrens W, Pohlabeln H, et al. Active and involuntary tobacco smoking and upper aerodigestive tract cancer risks in a multicenter case-control study. Cancer Epidemiol Biomarkers Prev. 2009:18:3353-61.

24. Thun MJ, Carter BD, Feskanich D, Freedman ND, Prentice R, Lopez AD, et al. 50-year trends in smoking-related mortality in the United States. N Engl J Med. 2013;368:351-64.

25. Swartz JB. Use of a multistage model to predict time trends in smoking induced lung cancer. J Epidemiol Community Health. 1992;46:311-5.

26. Flanders WD, Lally CA, Zhu BP, Henley SJ, Thun MJ. Lung cancer mortality in relation to age, duration of smoking, and daily cigarette consumption: results from Cancer Prevention Study II. Cancer Res. 2003;63:6556-62.

27. Knoke JD, Shanks TG, Vaughn JW, Thun MJ, Burns DM. Lung cancer mortality is related to age in addition to duration and intensity of cigarette smoking: an analysis of CPS-I data. Cancer Epidemiol Biomarkers Prev. 2004; 13:949-57.

28. Hazelton WD, Clements MS, Moolgavkar SH. Multistage carcinogenesis and lung cancer mortality in three cohorts. Cancer Epidemiol Biomarkers Prev. 2005;14:1171-81.

29. Meza R, Hazelton WD, Colditz GA, Moolgavkar SH. Analysis of lung cancer incidence in the Nurses' Health and the Health Professionals' Follow-Up Studies using a multistage carcinogenesis model. Cancer Causes Control. 2008; 19:317-28.

30. Wakai K, Marugame T, Kuriyama S, Sobue T, Tamakoshi A, Satoh H, et al. Decrease in risk of lung cancer death in Japanese men after smoking cessation by age at quitting: pooled analysis of three large-scale cohort studies. Cancer Sci. 2007:98:584-9.

31. Wakai K, Inoue M, Mizoue T, Tanaka K, Tsuji I, Nagata C, et al. Tobacco smoking and lung cancer risk: an evaluation based on a systematic review of epidemiological evidence among the Japanese population. Jpn J Clin Oncol. 2006;36:309-24

32. Katanoda K, Marugame T, Saika K, Satoh H, Tajima K, Suzuki T, et al. Population attributable fraction of mortality associated with tobacco smoking in Japan: a pooled analysis of three large-scale cohort studies. J Epidemiol. 2008:18:251-64.

33. Pope III CA, Burnett RT, Krewski D, Jerrett M, Shi Y, Calle EE, et al. Cardiovascular mortality and exposure to airborne fine particulate matter and cigarette smoke: shape of the exposure-response relationship. Circulation. 2009:120:941-8.

34. Ambrose JA, Barua RS. The pathophysiology of cigarette smoking and cardiovascular disease: an update. J Am Coll Cardiol. 2004:43:1731-7.

35. Wang C, Tu Y, Yu Z, Lu R. PM2.5 and Cardiovascular Diseases in the Elderly: An Overview. Int J Environ Res Public Health. 2015;12:8187-97.

36. Hackshaw AK, Law MR, Wald NJ. The accumulated evidence on lung cancer and environmental tobacco smoke. BMJ. 1997;315:980-8. 